\title{
La comunicación política en tiempos de crisis: una comparación entre Italia y España
}

\author{
Silvia SEMENZIN \\ silvia.semenzin@gmail.com \\ Universidad Complutense de Madrid
}

Recibido: 06/05/2015

Aceptado: 10/06/2015

\section{RESUMEN}

La época actual de crisis económica ha conllevado una crisis de tipo político también, que tiene como consecuencia una perdida de confianza por parte de los ciudadanos hacia las instituciones y los partidos tradicionales, que supuestamente actuarían como garantes de sus intereses. En Italia y España, dos países que se encuentran en situaciones parecidas, esto ha llevado al surgimiento de dos movimientos que han sabido explotar muchas de las herramientas que la comunicación política ofrece hoy en día, llegando a una considerable popularidad. Podemos y Movimento 5Stelle representan dentro de la comunicación política una autentica revolución y marcan para ella una era completamente nueva.

Palabras clave: Comunicación política, Movimento 5Stelle, Podemos, España, Italia.

\section{Political communication in the crisis age: a comparison between Italy and Spain}

\begin{abstract}
The present situation of economic crisis soon enough entailed a state of political crisis as well. Citizens are losing their faith in the political parties and the institutions who should safeguard them. When comparing Italy and Spain, it is easy to observe a number of similarities between the two countries: the economical crisis brought the birth of two political parties whose subtle and skillful exploitation of means of communication gave rise to their considerable popularity. Podemos and Movimento 5Stelle could be considered as representatives of a proper revolutionary approach to media and communication systems.
\end{abstract}

Keywords: Political Communication, Movimento 5Stelle, Podemos, Spain, Italy.

\section{Referencia normalizada}

Semenzin, S. (2015). "La comunicación política en tiempos de crisis: una comparación entre Italia y España”. Documentación de las Ciencias de la Información, Vol. 38. Páginas 83-102 


\section{INTRODUCCIÓN}

La crisis económica que atraviesa el mundo, y principalmente Europa, está suponiendo un cuestionamiento del propio Estado del Bienestar conllevando cada vez más una serie de contradicciones políticas y descontentos populares dentro de los estados.

Entre los países europeos que más están sufriendo estos dos tipos de crisis, económica y política, se colocan Italia y España, que ven agravada su situación por los continuos escándalos sobre corrupción y malgastar de dinero público que implican un alto nivel de desconfianza hacia los partidos políticos tradicionales, aparentemente incapaces de llevar a cabo soluciones prácticas para responder a las demandas ciudadanas.

La percepción ciudadana en los dos países es la de un alejamiento cada vez mayor del entorno político y sus representantes, que según la opinión publica más difundida utilizan el poder para lograr sus propios intereses personales en vez de actuar para los intereses públicos. Por estas razones, se puede observar como en ambos países durante las ultimas campañas electorales (en 2013 en Italia, actualmente en curso en España) han surgido dos movimientos políticos identificados como "de protesta", que pretenderían representar los intereses de los ciudadanos, contraponiéndose a los partidos tradicionales considerados demasiado obsoletos, corruptos y alejados de la realidad ciudadana. Estos movimientos son Movimento 5Stelle en Italia y Podemos en España.

En ambos casos, estos partidos emergentes han sido capaces de irrumpir con fuerza en la vida política de los países, ganandose mucha popularidad en la opinión publica y en el entorno político. Esto ha sido debido a la capacidad de ambos movimientos de utilizar de una forma inteligente e innovadora las herramientas de comunicación política, sobre todo los medios de comunicación y en particular los nuevos medios virtuales, con lo cual es frecuente llegar a considerar parecidos los dos partidos.

En realidad ellos se diferencian por la ideologías políticas y sobre todo por el tipo de comunicación política, con lo cual es interesante desarrollar un estudio comparativo entre los dos para entender porqué mientras en España Podemos va ganando en los sondeos electorales, en Italia Movimento 5Stelle, después del boom de las elecciones, ha ido quebrándose.

El estudio se centraría en las técnicas de comunicación política de los partidos, para ver las similitudes y diferencias y comprender las razones del suceso inicial tenido por los dos en la campaña electoral política, hasta analizar porqué en el caso de Movimento 5Stelle se podría afirmar que la comunicación política no ha sido suficiente para mantener el apoyo ciudadano.

Con el objetivo de desarrollar el trabajo de la mejor manera posible, he organizado el contenido en tres bloques: el primero ofrecería un marco teórico sobre lo que se entiende con el concepto central del estudio, es decir la comunicación política; el segundo englobaría en el estudio un ahondamiento sobre la crisis política actual, 
definiendo a través de varios autores dos de los problemas principales que afectan hoy en día a las instituciones italianas y españolas, es decir la corrupción y la perdida de confianza hacia las instituciones políticas y los partidos, para llegar a entender el surgimiento de los movimientos “de protesta”; el tercer y ultimo bloque estaría dedicado a presentar los dos movimientos políticos emergentes y sus técnicas de comunicación política a las que se debe su relevancia en el panorama político actual y a desarrollar un estudio comparativo entre los dos, para llegar a concluir que los dos movimientos se diferencian mucho más de lo que comúnmente se suele afirmar.

A través del estudio resulta interesante concluir que el papel de los nuevos medias van ganando importancia sobre los medios de comunicación tradicionales. Los dos partidos al final actúan como representantes de esta grande revolución que toca la comunicación política también, por esta razón he considerado interesante desarrollar este tipo de trabajo de investigación.

\section{LA COMUNICACIÓN POLÍTICA}

La comunicación política es la gran protagonista del objeto de estudio de este trabajo. Por ello, cabe empezar el estudio intentando dar una definición apropiada de lo que se entiende con el termino y comprender cuales son sus características principales.

El concepto está formado por otros dos conceptos muy amplios, es decir la comunicación y la política. De hecho muchos autores consideran fundamental disociar los dos términos y definirlos separadamente, pero en este trabajo solo se tomaran en consideración las definiciones que finalmente los autores llegan a dar de comunicación política. Sin embargo, es imprescindible remarcar que la comunicación política no es la política, si no que forma parte considerable del fenómeno político que se produce a través de la comunicación y los medios de comunicación de masas. Está claro además que uno de los momentos en que se desarrolla el máximo ejercicio de la comunicación política es durante las campañas políticas, sin embargo el fenómeno tiene una presencia constante dentro de todas las sociedades democráticas.

El estudio de la comunicación política implica cada vez mayor atención e investigación en la opinión publica, en el análisis de contenido de los mensajes, en las técnicas de propaganda y acción política, en los liderazgos y en el uso de los medios de comunicación y sus consecuentes efectos sociales. ${ }^{1}$

Diversos autores han señalado la dificultad de ofrecer una definición de la comunicación política. Entre la multitud de definiciones existentes, una de las más completas es la que propone la profesora Maria Jose Canel Crespo de la Universidad Complutense de Madrid, afirmando que la "comunicación política es la actividad de

\footnotetext{
${ }^{1}$ MORA LEDESMA, M., Metodología de la comunicación política en campañas electorales. México: Plaza y Valdés, 2011.
} 
determinadas personas e instituciones (políticos, comunicadores, periodistas y ciudadanos), en la que como resultado de la interacción, se produce un intercambio de mensajes con los que se articula la toma de decisiones políticas así como la aplicación de estas en la comunidad". ${ }^{2}$ En este caso la comunicación seria una forma de conexión entre instituciones políticas y comunidad para la toma de decisiones, que se articularía a través del lenguaje político. La misma autora acogía, en un libro precedente, una definición interesante elaborada por Trent y Friedenberg, donde la comunicación política representaría un conjunto de "símbolos creados por los medios de comunicación para que la sociedad mantenga la conciencia de la existencia de unas instituciones políticas; es decir, son las imágenes que ayudan a que el pueblo recuerde que se vota, que se controla al gobierno o que la constitución funciona”. ${ }^{3}$

Más interpretaciones las encontramos a través de una recolección de definiciones de varios autores en el libro "Comunicación política y opinión publica” de Ochoa" Chaffe la describe como "el papel de la comunicación en la política"; Blake y Haroldsen la ubican como "aquella que genera efectos sobre el funcionamiento de un estado político u otra entidad política"; Almon y Coleman la observan en cuanto "función básica que en cierto modo controla y hace posibles las demás funciones del sistema político". Meadow la reconoce como "el intercambio de símbolos o mensajes que, con un significativo alcance, han sido compartidos por, o tienen consecuencias para, el funcionamiento del sistema político". Fagen la supone como "una actividad comunicacional considerada política en virtud de las consecuencias actuales y potenciales que esta tiene para el funcionamiento del sistema político” y por último, el mismo Ochoa la define como aquel proceso de "transmisión y recepción de mensajes, desde y hacia los componentes del sistema político".

Es interesante observar como el conjunto de las definiciones afirman que la comunicación política tiene un fin especifico, es decir el fin de persuasión y convicción. Desde la época de Aristoteles, gobierna de hecho la visión de la comunicación como una búsqueda de los medios para persuadir.

Una de las características de este tipo de comunicación se refiere a las características de los mensajes políticos en la actualidad, en los cuales el uso de signos para llegar a persuadir se circunscribe al empleo meticuloso de símbolos, iconos y metáforas. Eso implicaría que el uso de la palabra tanto escrita como hablada ocuparía un segundo lugar en relación con el uso de elementos propios de la comunicación visual. ${ }^{5}$ Esto significaría también que la comunicación política pondría cada vez mayor relevancia en la forma, más que en el contenido de los mensajes.

\footnotetext{
${ }^{2}$ CANEL CRESPO, M.J., Comunicación Política. Madrid: Tecnos, 1999. p. 39.

${ }^{3}$ TRENT y FRIEDENBERG, 1995, en CANEL CRESPO, Madrid: Tecnos,1999. p. 20.

${ }^{4}$ Todos los autores citados en: OCHOA, O., Comunicación Política y Opinión Pública, México: McGraw Hill,2000. p. 3 y siguientes.

${ }^{5}$ DONDIS, D. La Sintaxis de la Imagen. Barcelona: Gustavo Gilli, 1997.
} 
La comunicación política contemporánea no puede prescindir de lo que son los medios de comunicación de masas, considerando la rápida evolución que estos han tenido hasta llegar a una considerable relevancia e influencia en la sociedad actual, caracterizada por la presencia constante de los nuevos medios en la vida de los individuos. Sobre esta base, damos una definición de comunicación política que nos muestra como "el arte de la retórica del discurso político clásico pero adaptado a un mundo con un nivel de desarrollo de los medios de comunicación como el contemporáneo en el que impera un alcance e inmediatez nunca antes vistos, y siempre con un fin: persuadir". 6

Sobre lo que se decía de la importancia que tiene la forma del mensaje político, llegando a superar el contenido, Manin observa que mientras antes "cada partido proponía a los electores un programa, que de llegar al poder, se encargaría de ejecutar, en el presente, la estrategia electoral de los candidatos y de los partidos se basa en la construcción de imágenes bastante vagas, en las cuales la personalidad de los líderes ocupa un lugar predominante, más que la promesa de medidas determinadas". ${ }^{7}$ Esto implicaría un lenguaje mucho más simple y etiquetado en la comunicación política (izquierda-derecha, bueno-malo,...), donde las ideologías y los programas políticos perderían cada vez más su importancia y adquirirían mayor relevancia en la opinión publica la figura del líder del partido, los slogans y los símbolos.

Se percibe entonces que la estrategia de acción de la política contemporánea, debe adecuarse a las reglas de los medios de comunicación de masas. Y no sólo ello, sino también "presentarse como novedosos, interesantes y llamativos, puesto que se debe competir con la oferta mediática habitual: es la lucha por ganar la atención (y el voto futuro) de gran parte de la sociedad". ${ }^{8}$

La comunicación política contemporánea parece ser en definitiva indisociable de los medios de comunicación de masas. La interacción entre la oferta de información política de los medios masivos y el comportamiento comunicativo del ciudadano representa una de las condiciones de la realidad de las sociedades democráticas modernas.

La exposición de las masas a los medios de comunicación resulta ser creciente gracias a las nuevas tecnologías y la revolución digital, donde la información y la inmensa cantidad de mensajes en circulación se han vuelto grandes protagonistas en la vida de las personas. En este panorama, los partidos políticos y los líderes se ven obligados cada vez más a servirse de las herramientas comunicativas que la contemporaneidad ofrece, donde los medios de comunicación son percibidos como instru-

${ }^{6}$ CORTES, R., La comunicación política como forma moderna de dominación: del discurso retórico al discurso icónico. Una aproximación critica. San Cristobal: Universidad de los Andes, 2010. p. 23.

${ }^{7}$ MANIN en DOS SANTOS, , 1992, ¿Qué queda de la representación política?, Caracas: Nueva Sociedad,1992. p. 9.

${ }^{8}$ CORTES, R. 2010, p. 25. 
mentos que permiten la transmisión de los mensajes políticos. En los próximos capítulos se analizaran ejemplos concretos de este uso novedoso que los partidos hacen en Italia y en España.

\section{LA CRISIS DE LA POLÍTICA}

La crisis económica actual que está afectando al mundo contemporáneo, se ha visto acompañada por una crisis de tipo político también, donde la desconfianza hacia las instituciones políticas y los partidos parece estar en continuo crecimiento entre los ciudadanos. Las instituciones democráticas se encuentran hoy en día debilitadas, generando un descontento en la población que cada vez menos se reconoce en sus representantes, afectando su interés y participación en la política. Si crisis política significa básicamente una crisis de la legitimidad de las instituciones que representan a los ciudadanos y falta de mecanismos de participación, el nivel de descontento, desconfianza y desaprobación, así como las tensiones y conflictos dentro de la sociedad (huelgas, protestas, movilizaciones populares, etc.) se vería aumentar directamente. ${ }^{9}$

La crisis económica ha sido responsable de la abertura de nuevas brechas sociales, dando forma a un incremento de las demandas de soluciones de los problemas que están afectando al Estado del Bienestar. La crisis mundial del sistema capitalista ha afectado de manera relevante los países del sur de Europa: Italia y España están entre esos países que más están sufriendo esta crisis.

El lento desmantelamiento del Estado del Bienestar a causa de la crisis económica, está afectando directamente a la credibilidad y calidad de la democracia, concepto que es tan conocido como complejo de entender. El termino “democracia” etimológicamente significa «poder del pueblo», pero si analizamos las obras de algunos autores muy importantes en el campo de las ciencias políticas encontraremos definiciones más completas para entender mejor el significado del término.

El autor Leonardo Morlino en el libro "Democracias y democratizaciones" (2003) ofrece cinco dimensiones del concepto de democracia, y entre ellas existe una definición mínima del concepto que puede indicar cuáles son los pocos aspectos, más inmediatamente controlables y esenciales a nivel empírico, que permiten establecer un umbral por debajo del cual un régimen no puede considerarse democrático. Según Leonardo Morlino, los regímenes se consideran democráticos si presentan al menos: sufragio universal; elecciones libres, competitivas, periódicas y limpias; mas de un partido; fuentes de información diferentes y alternativas. ${ }^{10}$

\footnotetext{
${ }^{9}$ Adaptado de PEREZ, J.I., La crisis política del Estado como quiebra de la legitimidad democrática en América Latina, 1994. Disponible en Internet: http://www.rieoei.org/oeivirt/rie04a03.htm .

${ }^{10}$ MORLINO, L. Democrazie e democratizzazioni, Bologna: Il Mulino, 2003. p. 8
} 
Según esta definición, Italia y España formarían parte de los países con un sistema democrático. Pero ¿cómo ha afectado la crisis a la calidad de la democracia?

Lo más relevante es que Morlino no se limita a dar unas definiciones técnicas de democracia, sino que nos ofrece una definición cualitativa de la misma también, es decir de lo que es una "buena democracia". En general, es un «ordenamiento institucional estable que mediante instituciones y mecanismos que funcionan correctamente consigue libertad e igualdad de los ciudadanos» y es «un régimen legitimado y estable que satisface a los ciudadanos». ${ }^{11}$

Varios autores de los ya mencionados coinciden en señalar la igualdad política como la base desde donde empieza la democracia. En relación a esto, hay cuatro dimensiones para evaluar la democracia: elecciones libres y justas, un gobierno abierto y que rinde cuentas, derechos civiles y políticos reconocidos y respetados y una sociedad cultural y estructuralmente democrática. Variables como la equidad formal entre partidos, la imparcialidad y transparencia de las instituciones, los derechos sociales y políticos, al igual que la participación ciudadana y su representatividad son piezas clave del sistema democrático, y su cumplimiento y respeto indicará su grado de calidad.

Es entonces cuando se puede establecer una correlación entre calidad democrática y corrupción, un problema que afecta los dos países de manera muy grave. Cuanta más calidad, mejor cumplimiento de los derechos y principios, lo que dificulta la corrupción política, es decir, a mayor calidad democrático menor grado de corrupción.

\subsection{La corrupción}

El fenómeno de la corrupción es un problema que está tocando mayormente los países de Europa meridional, entre los cuales se colocan España e Italia, generando una desconfianza crónica de los ciudadanos hacia las instituciones políticas.

Según el reportaje anual de 2014 de Transparency International ${ }^{12}$ que analiza los niveles de corrupción percibida en 175 países en el mundo, Italia es el primer país dentro de la clasificación europea (junto a Grecia y a Bulgaria) y está en la $69^{\circ}$ posición en la clasificación mundial, detrás de países como Sudáfrica y Kuwait, posicionándose así entre las naciones del mundo que no alcanzan ni un nivel de transparencia suficiente.

Por lo que concierne a España, esta se encuentra en la décima posición en la clasifica europea, ganando una mejor posición respeto al 2013, sin embargo sigue mante-

\footnotetext{
${ }^{11}$ MORLINO, L. p. 186-187.

12 Transparency International: Corruption perceptions index 2014. Web: http://www.transparency.org/ cpi2014/results .
} 
niendose entre esos países donde la corrupción afecta de forma considerable a la política.

Friedrich nos dice que «la corrupción existe cuando un responsable de un puesto público, con unas funciones y atribuciones definidas, es, por medios monetarios o de otra naturaleza, inducido a traicionar sus deberes y a actuar favoreciendo a quien proporciona el beneficio y, por ello, dañando al público y a sus intereses». ${ }^{13}$ Esta definición la podríamos encuadrar en la corrupción del sector público, ya que se busca favorecer a un grupo concreto así como la búsqueda de una política concreta o programa en lugar de buscar el beneficio común o general. Manuel Villoria Mendieta, da una definición bastante completa del concepto de corrupción, ya que dice que sería el tomar decisiones parciales por razones de beneficio monetario directo. También sería la elaboración de normas en las que se beneficia a un sector social sobre el conjunto de la comunidad a cambio de beneficios privados directos o indirectos. También sería corrupción pasar información de forma privilegiada a un actor individual o colectivo a cambio de algún tipo de recompensa, o cualquier acto que beneficie a una parte de la sociedad a cambio de sus votos, cuando el interés general sea dañado. ${ }^{14}$

Heidenheimer y Johnston dicen que «corrupción es cuando los plutócratas (miembros de la clase económica dominante) influyen sobre las decisiones y políticas públicas, cuando se hace meramente por interés privado, vía financiación de partidos o contribuciones a campañas». En este caso, la corrupción sería de normativa legal, es decir, de acuerdo con la legalidad. ${ }^{15}$

La visibilidad de las prácticas corruptas se ha hecho cada vez mayor. Los medios de comunicación diariamente nos informan de casos de corrupción o noticias relativas a ésta. Los de mayor calado son aquellos casos de corrupción que transmutan a escándalos, y son, por tanto, los más presentes en el día a día de la población española e italiana. Debido a esto, existe una sensación de desafección política e institucional en los ciudadanos españoles e italianos, en que se están apoyando los partidos emergentes, que utilizan cada vez más el tema del cambio político para ganar visibilidad entre la ciudadanía.

\subsection{La desconfianza en la política}

En la cultura política de los países democráticos se sigue apoyando mayoritariamente a las instituciones y valores de la democracia, aunque esta se vea afectada en su calidad y esto genere un extendido sentimiento de desconfianza hacia la política,

\footnotetext{
${ }^{13}$ VILLORIA MENDIETA, M., La corrupción política. Madrid: Editorial Síntesis, 2006. p. 38.

${ }^{14}$ VILLORIA MENDIETA, M. p. 40.

${ }^{15}$ Ibídem.
} 
los partidos y los políticos. Los cambios sociales debidos a la crisis económica y los problemas de corrupción han erosionado la identificación de los ciudadanos con los partidos, sin embargo, si nos basamos en las definiciones de los autores, los partidos políticos son considerados la columna vertebral de la democracias, pues constituyen las opciones en las que la población basa sus preferencias para ser gobernada.

Según la famosa definición de Weber el partido es "la forma de socialización que, descansando en un reclutamiento libre, tiene como fin proporcionar poder a su dirigente dentro de una asociación y otorgar por ese medio a sus miembros activos determinadas probabilidades ideales o materiales". ${ }^{16}$ Los partidos políticos surgen entonces como intermediarios entre la sociedad y el Estado y buscarían en principio un consenso y representatividad ciudadana.

A pesar de esto los partidos políticos tradicionales generan cada vez más frustración en los ciudadanos, revelándose incapaces de garantizar respuestas concretas a los problemas que afectan al país.

Cabe señalar en el estudio un fenómeno que desde hace tiempo afecta el sistema de partidos de España, es decir el fenómeno del bipartidismo perfecto. Para entender el concepto, un sistema bipartidista se define como aquel sistema donde dos partidos están en condiciones de igual competencia por la mayoría de los escaños y uno de estos dos partidos logra conseguir una mayoría suficiente para formar gobierno, y la existencia de terceros partidos carece de importancia en el grado en que no alteren una situación en que la participación en el poder estatal está reducida a solo los dos partidos principales. $^{17}$

El bipartidismo se ha vuelto problemático para la población española desde el momento en que la elección entre PP y PSOE ya no ha sido suficiente en la representación de la ciudadanía dentro de las instituciones políticas a causa de los múltiples escándalos de corrupción y derroche de dinero publico que han golpeado estos partidos, además de la incapacidad de llevar a cabo soluciones políticas y económicas convincentes.

El fenómeno se sumaria a las razones que nos harían entender porque un partido nuevo "de protesta" como Podemos está adquiriendo gran relevancia publica en la población, hasta llegar a representar un posible cambio dentro de la política española.

Por lo que concierne a Italia, el fenómeno del bipartidismo no existe, sin embargo se comparte con España esa desconfianza crónica hacia los representantes políticos, que desde muchos años se reparten el poder alejándose de los intereses públicos, con un continuo aumento de casos de mafia y corrupción que afectan al conjunto del país.

\footnotetext{
${ }^{16}$ WEBER, M., Economía y sociedad , Mexico: Fondo de Cultura Económica, 1969. p. 228.

${ }^{17}$ AGUILAR, M. Y GALINDO, L. Análisis comparativo de financiamiento a sistemas de partidos políticos en México, Argentina, Chile, Uruguay y Perú ( 2006). Disponible en Internet: http://catarina.udlap.mx/u_dl_a/tales/documentos/ladi/aguilar_t_md/capitulo_4.html .
} 


\section{LOS PARTIDOS EMERGENTES Y LA COMUNICACIÓN POLÍTICA}

Entremos ahora de lleno en el tema de la investigación, es decir el estudio comparativo de los dos partidos que han surgido en las ultimas elecciones en Italia y España. Hemos analizado las razones principales que están en el origen del nacimiento de estos partidos considerados "de protesta” para poder llegar al desarrollo de una investigación sobre las diferentes técnicas de comunicación política que han marcado un papel muy significativo para los dos partidos en la opinión publica. Entre las claves del éxito de ambos, se encuentra sobre todo una forma de entender las redes sociales e Internet en general como un canal fundamental en el ámbito de la comunicación política con la ciudadanía,. Para poder ampliar el estudio, he decidido analizar primero separadamente los dos partidos y después hacer la comparación.

\subsection{Movimento 5 Stelle}

El Movimento 5 Stelle es un partido político italiano, cuyo liderazgo es representado por el cómico Beppe Grillo. El partido se fundó en 2009 en Genova por Grillo y el "web guru" Gianroberto Casaleggio ${ }^{18}$, sin embargo el acto constitutivo del Movimiento se registró solo en diciembre de 2012.

El partido se caracteriza por utilizar un blog como referencia y herramienta básica de comunicación, a través del cual se promueven las iniciativas políticas y se pretende estimular una forma de democracia directa en contraposición con la democracia representativa. Una característica del movimiento es que desde siempre se ha propuesto como organización antipartidista y antideológica, tomando distancia de la clásica dicotomía izquierda-derecha. Las cinco estrellas del símbolo del partido representarían las temáticas principales en el programa político del movimiento, relativas a agua, medio ambiente, transporte, desarrollo y energía. ${ }^{19}$

En Octubre de 2012, unos meses antes de las elecciones políticas italianas, Grillo empezó una campaña política a través de su blog empezando a obtener atención publica y soporte en la red. La campaña se basaba principalmente en el rechazo de las fuerzas políticas vigentes en ese momento en Italia, acusados de ser ladrones y corruptos. El rechazo de las coaliciones caracterizará el movimiento hasta después de las elecciones, como ya veremos. Grillo estructuró su campaña política principalmente a través de su blog y de las redes sociales, desconfiando de los medios tradicionales

\footnotetext{
${ }^{18}$ BIORCIO R., NATALE, P.: Politica a 5 Stelle . Milano: Feltrinelli, 2013.

19 GRILLO, B. 4 ottobre: nasce un nuovo movimento (2009). Disponible en Internet: http://www.beppegrillo.it/2009/10/streaming_4_ott.html.
} 
también considerados corruptos, hasta llegar a prohibir a sus candidatos de participar en las tertulias televisivas o conceder entrevistas a los periódicos. ${ }^{20}$

El resultado fue que en las elecciones nacionales de febrero de 2013, el movimiento entró a formar parte de las mayores fuerzas políticas del país, obteniendo en breve tiempo un gran apoyo ciudadano, ganando un $25.6 \%$ de los votos a la Cámara de los Diputados y un 23.8\% en el Senado. El movimiento no llegó a ganar las elecciones, pero se clasificó como tercer partido en Italia, detrás del Partido Democrático de Bersani y el Pueblo de la Libertad de Berlusconi.

La novedad del éxito del partido de Grillo fue propiamente su estrategia de comunicación política, en una combinación de activismo online y ocupación de espacios públicos, a través de una confrontación directa con los ciudadanos. ${ }^{21}$ Hasta ese momento las fuerzas políticas dominantes en Italia habían ignorado las ventajas que podía ofrecer la revolución digital, observándola con escepticismo. De hecho, excepto para Movimento 5Stelle, la campaña nacional de 2013 fue organizada principalmente a través de los medias tradicionales (televisión y prensa).

La particularidad de Grillo fue poner énfasis en la web utilizada como una herramienta para la revolución política dentro de la sociedad italiana, llegando a crear el mito de la red como única posibilidad de democracia directa. Grillo y Casaleggio describen la web como transparente, coherente, con su propia lógica y alejada de cualquier tipo de corrupción, y que por lo tanto ofrece la posibilidad de cambiar los procesos políticos, sociales y organizativos. A diferencia de los partidos tradicionales, "la comunidad online se caracteriza por la solidaridad y la transparencia". ${ }^{22}$ Ademas Grillo afirma, a favor de la red, que "los ciudadanos que se sirven de la plataforma de internet están informados, los demás que utilizan la televisión y los periódicos están desinformados por el poder". 23

En conclusión el mito de la web llegaría a prometer a los ciudadanos una democracia directa y contrapuesta a la corrupta democracia liberal, la cual no permitiría esa participación.

\subsection{Podemos}

Podemos es un partido político español fundado en enero de 2014, cuyo secretario general es el profesor de Ciencias Politicas de la Universidad Complutense de Madrid, Pablo Iglesias Turrión. Cuatro meses después de su formación, Podemos participó en

\footnotetext{
${ }^{20}$ BORDIGNON, F., CECCARIN, L.: Five Stars and a cricket. Beppe Grillo shakes Italian politics. South European Society and Politics, 2013. p. 435

${ }^{21}$ CASTELLS, M. Networks of Outrage and Hope: Social Movements in the Internet Age. Cambridge, Polity Press, 2012.

${ }^{22}$ CASALEGGIO, G. y GRILLO, B., 2011 Siamo in guerra. Milano: Chiare Lettere, 2011. p. 46.

${ }^{23}$ CASALEGGIO, G., GRILLO, B., p. 10.
} 
las elecciones europeas de 2014 llegando al 7,9 de los votos y logrando cinco escaños, convirtiéndose en el cuarto partido más votado de España. Aunque rechace las etiquetas ideológicas, el programa político del partido presenta propuestas que se reconocen dentro de una ideología de izquierda radical. ${ }^{24}$ Es una de las estrategias comunicativa de Podemos no hablar ni de izquierda ni de derecha para intentar ser una "formación de amplio espectro ideológico y con las menos aristas posibles" 25 , sin embargo la plataforma política de Podemos se basa en la conservación del medioambiente, la recuperación del déficit democrático y la lucha contra de las grandes empresas, los bancos y la economía neoliberal. A parte, en el programa se prevén incentivos a la pequeña empresa, a la producción de comida a nivel local, al transporte publico, y se acoge también la propuesta de una nacionalización de la mayoría de los servicios públicos, con lo cual las propuestas encajan en una ideología de izquierda.

De acuerdo con los últimos sondeos sobre los posibles resultados de las elecciones políticas previstas para mayo de 2015, el partido ganaría las elecciones con un $27 \%$ de los votos, situándose como mayor fuerza política en España, superando los partidos tradicionales (PP y PSOE) y acabando con el bipartidismo. Si estas predicciones se confirmasen reales, marcarían sin duda un fin de ciclo en la política española.

$\mathrm{Al}$ igual que Movimento 5Stelle, el rápido éxito de Podemos se debe a una estrategia comunicativa basada en un amplio uso de Internet y de los medios audiovisuales. Además de la nueva comunicación a través de la red y de las redes sociales, Podemos se sirve mucho de los medios tradicionales, la televisión en primer lugar. En esta estrategia cabe nombrar "la Tuerka”, el programa de debate político que comenzó a emitirse hace algunos años en la televisión vallecana Tele-K, que tiene una importancia central. "La Tuerka" fue desde el principio el laboratorio en el que Pablo Iglesias, Íñigo Errejón o Juan Carlos Monedero, entre otros, aprendieron cómo transmitir mensajes políticos en el medio audiovisual. ${ }^{26}$

El éxito de Iglesias es sobre todo producto de un equipo, vinculado al activismo de izquierdas y a la Facultad de Ciencias Políticas de la Universidad Complutense de Madrid, que orienta sus esfuerzos a comunicar, es decir pensar no solo en el contenido del mensaje, si no sobre todo en su forma. El resultado de la creación audiovisual de Podemos es un saldo muy elevado: 86 vídeos subidos al canal en Youtube desde la presentación de la candidatura, sumados a los subidos por los canales de La Tuerka o Fort Apache y otros círculos.

24 PUCCIARELLI, Podemos, la sinistra spagnola oltre la sinistra, 2014. Web: http://ilmegafonoquotidiano.it/news/podemos-la-sinistra-spagnola-oltre-la-sinistra .

${ }^{25}$ GAREA, F.: Podemos supera a PSOE y PP y rompe el tablero electoral. 2014. Disponible en Internet: http://politica.elpais.com/politica/2014/11/01/actualidad/1414865510_731502.html .

${ }^{26}$ MURIEL, E. Cinco claves del éxito de la campaña electoral de Podemos, 2014. Disponible en Internet: http://www.lamarea.com/2014/05/26/cinco-claves-del-exito-de-la-campana-electoral-de-podemos/ . 
Tras el triunfo en las elecciones europeas, el alcance de la figura de Pablo Iglesias se ha multiplicado. Hoy, todos los medios quieren hablar con él y reproducir sus declaraciones, consiguientemente Iglesias se ha vuelto omnipresente dentro de los medios de comunicación.

Por lo que concierne a la red, Podemos utiliza su propia página de internet y las redes sociales, principalmente Facebook, Twitter y Youtube, para lanzar iniciativas políticas, votos y elaborar una política "trasversal, transparente y transformadora"27, promoviendo como en el caso de Movimento 5Stelle la democracia directa y el mito de la red.

Según destaca el periódico El País, Iglesias ya es "el político más influyente en Europa en redes sociales”28. Según el software de comunicación de Augure, Pablo Iglesias fue el candidato que contó con más influencia digital durante la campaña electoral.

En Twitter cuenta con 388.000 seguidores frente a los 172.000 del PSOE y los 169.000 del PP. Su página en Facebook cuenta con casi 800.000 seguidores, muy por encima de los 72.000 de los socialistas y los 76.000 de los conservadores. ${ }^{29}$

\subsection{Un estudio comparativo}

Entremos ahora de lleno en el estudio inicialmente propuesto, es decir un análisis de las similitudes y diferencias entre dos partidos que basan su estrategia política en la comunicación, pero entendiendo porque mientras uno va ganando preferencias en los sondeos y encuestas, el otro parece estar a un paso de romperse.

Como hemos visto hay muchas similitudes entre los dos partidos, con lo cual es muy común llegar a plantearse una comparación. En primer lugar los dos movimientos han basado gran parte de su actividad política en colocarse como contrapuestos a los partidos políticos tradicionales, considerados corruptos y ladrones. En segundo lugar, ambos movimientos se han estructurado alrededor de un líder mediatico, y por ultimo, ambos partidos han hecho un masivo uso de las nuevas herramientas de comunicación, explotando las ventajas ofrecidas por Internet y las redes sociales. Pero aunque en un primer impacto las similitudes parecen ser varias, hay que dar un enfo-

\footnotetext{
${ }^{27}$ CARRILLO, F.: Las técnicas de comunicación de Pablo Iglesias y Podemos: La revolución empieza con palabras, 2014. Disponible en Internet: http://www.topcomunicacion.com/noticia/5656/estrategiade-comunicacion-depablo-iglesias-podemos-comunicacion-politica\#.

${ }^{28}$ GONZALO, P. Podemos y Pablo Iglesias: un caso de estudio sobre el poder amplificador de las redes sociales y la participación ciudadana, 2014. Disponible en Internet: http://www.periodismociudadano.com/2014/06/02/podemos-y-pablo-iglesias-un-caso-de-estudio-sobreel-poderamplificador-de-las-redes-sociales-y-la-participacion-ciudadana/

${ }^{29}$ MACHUCA, P.: Podemos: Nuevas herramientas para una nueva política, 2014. Disponible en Internet: http://www.huffingtonpost.es/2014/10/22/podemos-herramientas-comunicacion_n_6020650.html.
} 
que más profundo a las diferencias que los dos movimientos presentan, porque esas diferencias son las que marcan una neta distancia entre los partidos.

La primera diferencia la encontramos en la estructura : Movimento 5Stelle nace alrededor de un líder mediatico, mientras que Podemos nace por un equipo de profesores, que se ha dedicado luego a la construcción de un líder. ${ }^{30}$

Diferentemente de Movimento 5Stelle, Podemos se caracteriza por una democracia en red muy avanzada, donde las decisiones y los congresos representan verdaderos debates, sujetos luego a votación en un social forum (un asamblea de ciudadanos online). La elección de sus candidatos, de sus programas y de sus estrategias organizativas y políticas se han decidido, desde el nacimiento de la formación, a través de votaciones principalmente desarrolladas por Internet. ${ }^{31}$ Por otro lado, Movimento 5Stelle mantiene una estructura más rígida, donde la red representa un instrumento de participación y consulta, pero de hecho las políticas y estructuras del Movimiento siguen siendo controlados y definidos por Grillo y Casaleggio. ${ }^{32}$

Sobre lo que concierne a la democracia interna de los movimientos, esta marca otra diferencia básica entre los movimientos. Sobre este tema, me refiero a la lógica de la expulsión característica de Movimento 5Stelle, que se ha revelado finalmente dañina para el. Después de las elecciones de 2013, el movimiento ha sido caracterizado por varios desacuerdos entre los candidatos por razones ideológicas, llevando Grillo a expulsar a algunos de los miembros del partido. Esto ha levantado numerosas criticas sobre la falta de democracia interna al partido y ha causado una ingente perdida de apoyo entre los sostenedores. ${ }^{33}$

Otra diferencia la encontramos dentro de las propias técnicas de comunicación política y al diferente uso de los canales comunicativos para el alcance de los objetivos. El éxito de Podemos se debe a una inteligente estrategia de triangulación territoriored - televisión. Podemos ha sabido explotar la red, pero sin rechazar los medios tradicionales; su constante presencia en las tertulias televisivas y en los periódicos le ha permitido llegar a un publico más amplio, a través de la continua búsqueda de choques dialécticos con políticos y periodistas. Se puede decir que Podemos utiliza el territorio para la participación, la red para la conexión y la televisión para la persuasión. $^{34}$

En cambio, Movimento 5Stelle en la campaña política de 2013 llegó a rechazar cualquier tipo de colaboración con periodistas y negó la confrontación con las demás

\footnotetext{
${ }^{30}$ FAgGiOnAtO, G.: Podemos, Luca Raffini: una lezione per Grillo, 2014. Entrevista disponible en Internet: http://www.lettera43.it/esclusive/podemos-luca-raffini-una-lezione-per-grillo_43675146608.htm .

${ }^{31}$ MACHUCA, P. 2014.

${ }^{32}$ FAGGIONATO, G., 2014.

${ }^{33}$ NATALE, S. y BALLATORE, A.: The web will kill them all: new media, digital utopia, and political struggle in the Italian 5-Star Movement . 2014. p. 116.

${ }^{34}$ FAGGIONATO, G., 2014.
} 
fuerzas políticas. Esto ha llevado cada vez más a los ciudadanos italianos a una toma de conciencia de la falta de argumentaciones de Grillo y sus seguidores. Mientras Iglesias marca con evidencia sus conocimientos del lenguaje audiovisual y de las técnicas de oratorias a través de unas conversaciones con tono pausado y razonado ${ }^{35}$, Grillo cae a menudo en la trampa de un lenguaje a gritos, vulgar y ofensivo. ${ }^{36}$

Otra diferencia para remarcar, se sitúa dentro de la propia connotación política de los dos movimientos. Mientras Podemos, no obstante rechace las etiquetas de izquierda radical, se coloca dentro a una ideología principalmente de izquierda, Movimento 5Stelle siempre ha tenido una connotación política ambigua y poco clara. Especialmente después del boom de las elecciones de 2013, el movimiento ha ido perdiendo coherencia en el mensaje y consiguiente relevancia política ${ }^{37}$ Muchas de las declaraciones de Grillo, sobre todo en el ano 2014, se colocaban dentro de un cuadro de declaraciones pertenecientes a ideologías de extrema derecha, en particular con referencia a temas concernientes a los inmigrantes, la mafia y la gestión de la economía, con lo cual el descontento popular ha sido evidente sobre todo en las elecciones municipales de 2013, cuando Movimento 5Stelle resultó perder casi la mitad de los votos. Las posiciones tomadas en los últimos tiempos por el movimiento italiano resultan ser tendencialmente xenófobas, mientras Podemos acogería los valores de tolerancia, solidaridad y acogimiento provenientes de la izquierda. ${ }^{38}$

Después de las elecciones, Movimento 5Stelle ha perdido mucho apoyo por no haber sido capaz de llevar a cabo ninguna de las propuestas políticas dentro del programa inicial, excepto la abolición de la ley Lodo Alfano. ${ }^{39}$ Esto es causado por el rechazo de cualquier tipo de coalición con los otros partidos, llevando el movimiento a una perdida de sentido dentro de la gestión del estado y la consiguiente pérdida de credibilidad entre los sostenedores. Podemos por su parte no excluiría la posibilidad de alianzas con otras fuerzas de izquierda, con las cuales podría encontrarse una convergencia programática.

En conclusión, Movimento 5Stelle ha sabido explotar la fuerza de la red pero no ha sabido llegar a formular un programa político concreto y coherente después de haber ganado visibilidad, con lo cual va perdiendo apoyo en la opinión publica, según las encuestas y las últimas elecciones municipales.

\footnotetext{
${ }^{35}$ MAESTRE, A. Podemos y la comunicación política, 2014. Disponible en Internet: http://www.eldiario.es/agendapublica/nueva-politica/nuevo-sistema-partidos-Espana_0_324068391.html . ${ }^{36}$ NATALE, S. y BALLATORE, A. p. 111.

${ }^{37}$ NATALE, S. y BALLATORE, A. p. 120.

${ }^{38}$ FAGgiONATO, G.: Podemos, Luca Raffini: Una lezione per Grillo, 2014. Entrevista disponible en Internet: grillo_43675146608.htm .

${ }^{39}$ Vease el programa de Movimento 5Stelle en la pagina web del partido, donde están marcadas con un "tic" todas las propuestas políticas llevadas a cabo.
} 
Podemos parece tener una gran diferencia en términos de propuestas y programas políticos. Aunque muchas de las iniciativas que se quieren llevar a cabo dentro del programa electoral sean objeto de denuncia por muchos, por ser inaplicables y populistas (por ejemplo, las propuestas sobre el rédito de ciudadanía o la tasación de las empresas privadas extranjeras), el programa aparenta en general calidad y es un estudio razonado y concreto de las propuestas formulado por el equipo académico, apoyandose en una base ideológica socialdemócrata . Ese uso de un discurso sencillo y que apela a la emoción se debe al intento de "traducir esos diagnósticos de la izquierda, ya que uno de los dramas de los movimientos socialistas y del marxismo ha sido su incapacidad para traducirse", ${ }^{40} \mathrm{e}$ s decir el constante intento de llegar a "la gente".

Finalmente, se podría decir que Podemos ha incorporado muchas de las demandas que proponía el movimiento de los Indignados, como por ejemplo el fin de los altos sueldos de los políticos o las primarias abiertas, añadiendo novedades a su programa en términos de gestión económica y política.

\section{CONCLUSIONES}

Durante el desarrollo del trabajo ha quedado claro que la situación política actual en que se encuentran Italia y España es muy parecida y ha llevado a la luz el nacimiento de dos movimientos que a primera vista se parecen mucho. Sin embargo, el estudio sobre las diferentes técnicas de comunicación utilizadas por los dos partidos y las propuestas políticas llevan a la conclusión que en realidad las similitudes son muchas menos que las diferencias substanciales que los caracterizan.

A pesar de eso, los dos movimientos representan un buen ejemplo de como la retórica del cambio político y la retórica de la revolución digital pueden unirse para formar un discurso único y coherente, que mande un mensaje positivo a los potenciales votantes.

En este sentido hay que reconocer que la revolución de estos partidos empieza con la comunicación política y conlleva una reflexión sobre el cambio que los nuevos medios están ofreciendo en todos los ámbitos de la comunicación. Sin embargo, habría que reconocer también que el resultado que este tipo de revolución en la comunicación política está teniendo, es que el lenguaje se vuelve cada vez más simple y "vago", y la forma del mensaje va ganando cada vez más relevancia que el contenido.

Por lo que concierne a la pregunta inicial del porqué Podemos parece estar teniendo más éxito que Movimento 5Stelle me permito contestar con unas reflexiones

\footnotetext{
${ }^{40}$ MURIEL, Eduardo: Cinco claves del éxito de la campaña electoral de Podemos, La Marea (2014). Disponible en Internet: http://www.lamarea.com/2014/05/26/cinco-claves-del-exitodela-campana-electoral-de-podemos/.
} 
personales: por lo visto, el discurso de Podemos no se queda en una critica anti-casta, si no que propone un programa electoral más razonado, a diferencia de Movimento 5Stelle que cada vez más se queda en lo que parece ser poco más que abstracta y confusa demagogia. Por esta razón, las herramientas comunicativas utilizadas por Movimento 5Stelle no llegan a ser suficientes, porque su mensaje político es cada vez más vacío y confuso. Aunque parece ser verdad que la forma va ganando sobre el contenido, el contenido (afortunadamente) sigue teniendo su importancia, por lo que los votantes de Movimento 5Stelle piden respuestas más concretas y especificas, teniendo en cuenta que el peso político que ha tenido el partido después de las elecciones ha sido muy poco significativo.

En conclusión, se podría afirmar que una buena comunicación política hoy en día constaría en saber utilizar los medios de comunicación tradicionales juntos con los nuevos, sin descartar herramientas como la televisión - que todavía sigue siendo una gran presencia en la vida de los individuos - y los periódicos. En este sentido, un buen estratega de comunicación política no debería olvidarse de la importancia de la cercanía con la población, para llenar esos vacíos dejados por las fuerzas políticas tradicionales, lo cual puede ser obtenido a través de los medios y a través del caracara en los territorios. Los dos partidos han sido capaces de llegar a una amplia y heterogénea parte de la población, utilizando estas estrategias y posicionándose de alguna manera "más allá” de las ideologías. 


\section{BIBLIOGRAFÍA}

BIORCIO Roberto, NATALE, Paolo.: Politica a 5 Stelle. Milano, Feltrinelli, 2013.

CANEL CRESPO, Maria José: Comunicación Política. Madrid, Tecnos, 1999.

CANEL CRESPO, Maria José: Comunicación Política, una guía para su estudio y práctica, Madrid, Tecnos. 2006.

CASALEGGIO, Gianroberto, GRILLO Beppe: Siamo in guerra. Milano, Chiare Lettere, 2011.

CASTELLS, Manuel: Networks of Outrage and Hope: Social Movements in the Internet Age. Cambridge, Polity Press, 2012.

CORTES, Reinaldo: La comunicación política como forma moderna de dominación: del discurso retórico al discurso icónico. Una aproximación critica. San Cristobal, Universidad de los Andes, 2010.

DONDIS, Donis: La Sintaxis de la Imagen. Barcelona, Gustavo Gilli, 1997.

DOS SANTOS, Mario: ¿Qué queda de la representación política?, Caracas, Nueva Sociedad, 1992.

MORA LEDESMA, Martín: Metodología de la comunicación política en campañas electorales, Mexico, Plaza y Valdés, 2011.

MORLINO, Leonardo: Democrazie e democratizzazioni, Bologna, Il Mulino, 2003.

OCHOA, Oscar: Comunicación Política y Opinión Pública. México, McGraw Hill, 2000.

VILLORIA MENDIETA, Manuel: La corrupción política. Madrid, Editorial Síntesis, 2006.

WEBER, Max, Economia y sociedad, Mexico, Fondo de Cultura Económica, 1969.

\section{ARTICULOS DE REVISTAS}

BORDIGNON, Fabio, CECCARINI, Luigi: Five Stars and a cricket. Beppe Grillo shakes Italian politics. South European Society and Politics, Vol. 18, ํ 4, 2013, pp. 427-449.

NATALE, Simone, BALLATORE, Andrea: The web will kill them all: new media, digital utopia, and political struggle in the Italian 5-Star Movement. Media, Culture \& Society, Vol. N 1, 2014, pp. 105-121.

\section{WEBGRAFÍA}

AGUILAR TORRES, María y GALINDO HERNÁNDEZ, Lariza: Análisis comparativo de financiamiento a sistemas de partidos políticos en México, Argentina, Chile, Uruguay y Perú (2006). Disponible en Internet: http://catarina.udlap.mx/u_dl_a/tales/documentos/ladi/aguilar_t_md/capitulo_4.ht $\mathrm{ml}$ \#.[Enero de 2015].

BARRIO, Astrid: ¿Por qué se dice que Podemos puede acabar con el bipartidismo?, 
El Diario (2014). Disponible en Internet:

http://www.eldiario.es/agendapublica/nuevapolitica/ nuevo-sistema-partidosEspana_0_324068391.html. [6 de enero de 2015]

CARRILLO, Fran: Las técnicas de comunicación de Pablo Iglesias y Podemos: La revolución empieza con palabras, La Fabrica de Discursos (2014). Disponible en Internet: http://www.topcomunicacion.com/noticia/5656/estrategia-decomunicacion-depabloiglesias-podemos-comunicacion-politica\#. [5 de enero de 2015].

FAGGIONATO, Giovanna: Podemos, Luca Raffini: una lezione per Grillo, Lettera 43 (2014). Entrevista disponible en Internet: http://www.lettera43.it/esclusive/podemos-lucaraffini-una-lezione-pergrillo_43675146608.htm [Diciembre 2014-enero 2015].

GAREA, Fernando: Podemos supera a PSOE y PP y rompe el tablero electoral. El Pais (2014). Disponible en Internet: http://politica.elpais.com/politica/2014/11/01/actualidad/1414865510_731502.htm l. [7 de enero de 2015].

GONZALO, Paula: Podemos y Pablo Iglesias: un caso de estudio sobre el poder amplificador de las redes sociales y la participación ciudadana, Periodismo Ciudadano (2014). Disponible en Internet: http://www.periodismociudadano.com/2014/06/02/podemos-y-pablo-iglesias-uncasodeestudio-sobre-el-poder-amplificador-de-las-redes-sociales-y-laparticipacion-ciudadana/ [5 de enero de 2015].

GRILLO, Beppe: 4 ottobre: nasce un nuovo movimento (2009). Disponible en Internet: http://www.beppegrillo.it/2009/10/streaming_4_ott.html. [Diciembre 2014-enero 2015].

GRILLO, Beppe: Beppe Grillo e la Carta di Firenze, Blog di Beppe Grillo (2009). Disponible en Internet: http://www.beppegrillo.it/2009/04/la_carta_di_fir.html. [5 de enero de 2015].

GRILLO, Beppe: Programma del Movimento 5 Stelle, Blog di Beppe Grillo (2012), Disponible en Internet: http://www.beppegrillo.it/iniziative/movimentocinquestelle/ProgrammaMovimento-5-Stelle.pdf. [3 de enero de 2015].

Il Post: Che cos'è Podemos (2014), Disponible en Internet: http://www.ilpost.it/2014/11/02/podemos-spagna/ [5 de enero de 2015].

MACHUCA, Pablo: Podemos: Nuevas herramientas para una nueva política, Huffington Post (2014). Disponible en Internet: http://www.huffingtonpost.es/2014/10/22/podemosherramientas/comunicacion_n_ 6020650.html. [5 de enero de 2015].

MAESTRE, Agapito: Podemos y la comunicación política, El Mundo (2014). Disponible en Internet: http://www.elmundo.es/opinion/2014/11/30/547b82deca47413a3f8b4577.html. [6 de enero de 2015]. 
MURIEL, Eduardo: Cinco claves del éxito de la campaña electoral de Podemos, La Marea (2014). Disponible en Internet: http://www.lamarea.com/2014/05/26/cincoclaves-del-exitode-la-campana-electoral-de-podemos/. [5 de enero de 2015].

Pagina web de Podemos, http://podemos.info/. [Enero de 2015].

PEREZ, Jesús Ignacio: La crisis política del Estado como quiebra de la legitimidad democrática en América Latina. Revista Iberoamericana de Educación (1994). Disponible en Internet: http://www.rieoei.org/oeivirt/rie04a03.htm [Diciembre de 2014].

PUCCIARELLI, Matteo: Podemos, la sinistra spagnola oltre la sinistra, MicroMega (2014). Disponible en Internet: http://ilmegafonoquotidiano.it/news/podemos-la sinistraspagnola-oltre-la-sinistra [4 de enero de 2015].

Repubblica: Movimento 5 stelle, consenso dimezzato. (2013) Disponible en Internet: http://www.repubblica.it/speciali/politica/elezioni-comunalirisultati2013/ 2013/05/27/news/cinquestelle-59749409/ [4 de enero de 2015].

TONIUTTI, Tiziano: Risultati elezioni, boom Movimento 5 Stelle. Repubblica (2013). Disponible en Internet:

http://www.repubblica.it/speciali/politica/elezioni2013/2013/02/25/news/elezioni_ grillo_m5s_risultati_reazioni-53396877/. [7 de enero de 2015].

Transparency International: Corruption perceptions index 2014. Web: http://www.transparency.org/cpi2014/results [Enero de 2015 